\title{
An Integrated Bidirectional Multi-Channel Opto- Electro Arbitrary Waveform Stimulator For Treating Motor Neurone Disease
}

\author{
Dai Jiang ${ }^{1}, \mathrm{Yu} \mathrm{Wu}{ }^{1}$, Noora Almarri ${ }^{1}$, Maryam Habibollahi ${ }^{1}$, Fangqi Liu ${ }^{1}$, J. Barney Bryson ${ }^{2}$, Linda Greensmith ${ }^{2}$, \\ and Andreas Demosthenous ${ }^{1}$

\begin{abstract}
${ }^{1}$ Department of Electronic and Electrical Engineering, University College London, London WC1E 7JE, UK
d.jiang@ucl.ac.uk, a.demosthenous@ucl.ac.uk
\end{abstract} \\ ${ }^{2}$ Department of Neuromuscular Diseases, UCL Institute of Neurology, Queen Square, London WC1N 3BG, UK
}

\begin{abstract}
This paper presents a prototype integrated bidirectional stimulator ASIC capable of mixed opto-electro stimulation and electrophysiological signal recording. The development is part of the research into a fully implantable device for treating motor neurone disease using optogenetics and stem cell technology. The ASIC consists of 4 stimulator units, each featuring 16-channel optical and electrical stimulation using arbitrary current waveforms with an amplitude up to $16 \mathrm{~mA}$ and a frequency from $1.5 \mathrm{~Hz}$ to $50 \mathrm{kHz}$, and a recording front-end with a programmable bandwidth of $1 \mathrm{~Hz}$ to $4 \mathrm{kHz}$, and a programmable amplifier gain up to $74 \mathrm{~dB}$. The ASIC was implemented in a 0.18-um CMOS technology. Simulated performance in stimulation and recording is presented.
\end{abstract}

Keywords-Electrophysiological recording, integrated circuits, motor neurone disease, optogenetics, opto-electro stimulation.

\section{INTRODUCTION}

Motor neurone disease (MND) destroys the motor neurons that control skeletal muscle activities, which severely limits the patient's motor function and independence in daily life, and dramatically shortens life expectancy. A 2016 survey shows globally 330,918 individuals had MND [1]. Currently there is no cure or effective therapy for MND. Regenerating the damaged motor nerve using stem cellderived motor neurons shows potential of reversing the paralysis caused by MND, but the stem cell motor neurons must be engrafted close to the target muscles to ensure muscle reinnervation before muscle atrophy occurs and paralysis becomes irreversible, given the slow rate of nerve growth of $\sim 1 \mathrm{~mm} /$ day. Therefore, reconnecting the motor neural circuits to the central neural system becomes extremely challenging. Instead, the regenerated motor neurons can be stimulated locally to facilitate coordinated locomotion. A recent study with rodents has demonstrated controllable muscle contraction under optical stimulation, after paralyzed muscles were innervated with engrafted stem cell motor neurons genetically modified to express the light-sensitive ion channel channelrhodopsin-2 (ChR2) [2]. This discovery laid the foundation of treating muscle paralysis caused by MND with therapies combining electronics, optogenetics and stem cell technologies, to deliver intervention at a cellular level to individual motor units.

A research platform device is needed for extensive in vivo animal studies to ensure successful clinical translation of this therapeutic strategy. A conceptual diagram of such a device is

This work was supported by the Medical Research Council UK under grant MR/R011648/1.

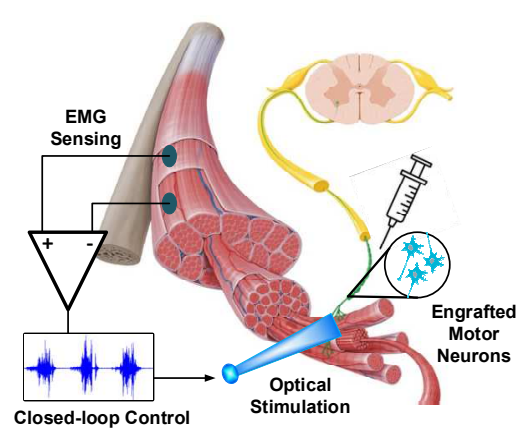

Fig. 1. Conceptual diagram of closed-loop optogenetic stimulation for treating motor neurone disease using stem cell-derived motor neurons.

illustrated in Fig. 1, where stem cell-derived motor neurons expressing ChR2 are engrafted close to the muscle innervation points. Optical stimulators excite the regenerated motor nerve to facilitate muscle contraction, which is monitored by sensing the electrophysiological response such as electromyography (EMG), where personalized optimal closed-loop stimulation strategy can be derived. There are four key specifications for such a device: 1) It should provide multi-channel stimulation and electrophysiological recording for coordinated muscle movement since the stimulation is localized to individual muscles; 2) It should provide multiple electrophysiological recording modality, including EMG and neural response to the stimulation; 3) Multi-mode stimulation strategy may be beneficial because evidence in animal studies shows that electrical stimulation can promote stem cell nerve rehabilitation [3] and hybrid optogenetic and electrical stimulation improves stimulation outcome [4]; 4) The device should be fully implantable to reduce the risk of infection and to provide chronical stimulation and monitoring, since muscle innervation from the stem cell motor neurons takes 30-60 days after engraftation [1].

The majority of optogenetic devices reported in literature require percutaneous connection to an external device. Recent research efforts have seen development in integrated optical stimulators towards fully implantable optogenetics devices. However, most of the reported design target optogenetic applications are in the brain, where electrophysiological sensing is primarily limited to neural recording. The ASICs reported in [5-7] provide optical only stimulation with recording units for action potentials and local field potentials, while the design in $[8,9]$ provide both optical and electrical stimulation but without sensing capability. The device in [10] targets the peripheral nerves but only supports single direction opto-electro stimulation.

*Background cartoon of muscle fiber and motor nerve is from https://www.kenhub.com/en/library/anatomy 


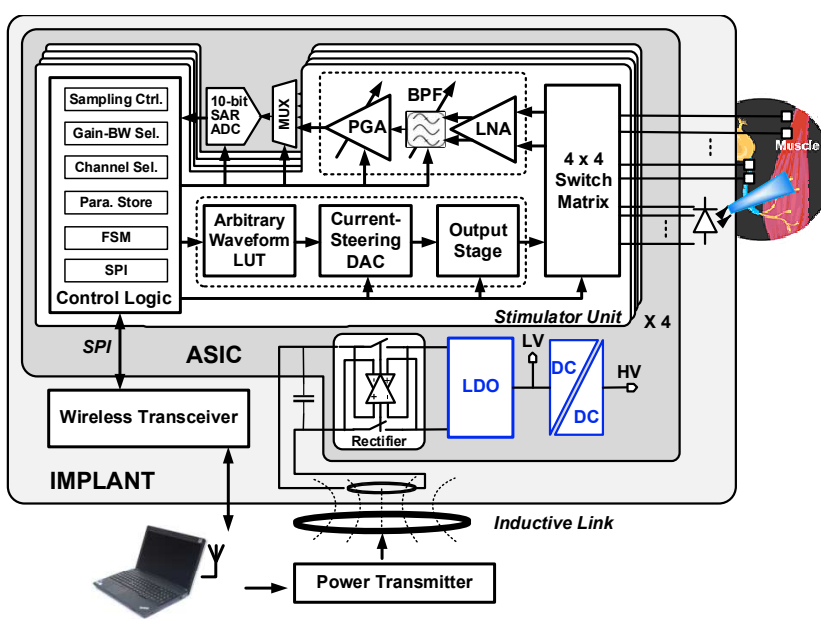

Fig. 2. System diagram of the proposed bidirectional opto-electro

This paper presents a CMOS system-on-chip (SoC) design of a bidirectional stimulator ASIC capable of both optical and electrical stimulation and electrophysiological recording on up to 64 channels ( $4 \times 4 \times 4)$. It is developed as part of a fully implantable opto-electro stimulator for the investigation on using optogenetics and stem cell technology in small animals to treat MND. The remainder of this paper is organized as follows. Section II describes the design and implementation of the bidirectional stimulator ASIC. Section III presents the simulated performance of the ASIC, and concluding remarks are drawn in Section V.

\section{SYSTEM IMPLEMENTATION}

\section{A. System Architecture}

The proposed architecture of the implant is illustrated in Fig. 2. The implant consists of the bidirectional stimulator ASIC, 470-nm blue micro-LED arrays for optical stimulation, electrode arrays for electrical stimulation and electrophysiological recording, a wireless transceiver for communicating with the implant during operation, and the receiving side of an inductive link for wireless power transfer [11]. The ASIC includes four parallel stimulator units and a power management unit. The stimulator units can operate individually or work together for synchronized stimulation. Each stimulator unit consists of a current driver capable of generating arbitrary current waveforms for driving the LEDs or electrodes, and a recording front-end with 3-stage programmable amplification and filtering, where all the stimulator units share a 10-bit SAR ADC for digitizing the recorded electrophysiological signals of interest. Stimulation and recording are multiplexed via a switch matrix connected to 4 anode and 4 cathode contacts. The embedded control logic on each stimulator unit manages its operation.

\section{B. Control Logic}

The implant communicates with the external computer via a wireless radio link. Commands containing parameters for stimulation and recording, and IDs of the chosen stimulator unit, are received by the wireless transceiver and broadcasted via a serial peripheral interface (SPI) to all the stimulator units. The control logic in the stimulator unit with the matching ID stores the parameters and triggers a finite-state machine (FSM). Operating under a $100 \mathrm{kHz}$ master clock, the FSM repeatedly loads the stored parameters at the beginning of each

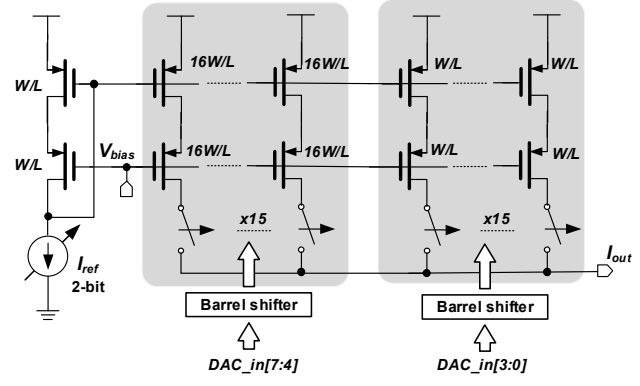

(a)

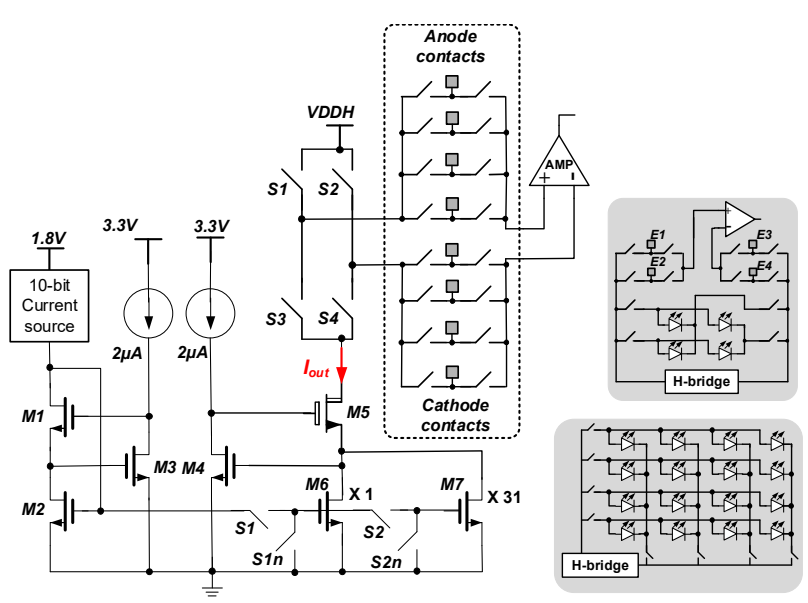

(b)

Fig. 3. Implementation of the current driver: (a) Schematic of the 10-bit current source; (b) Schematic of the output stage of the current driver and the $4 \times 4$ switch matrix. Two configuration examples of the LED/electrode connection are shown in the shaded areas.

current pulse cycle, so that the throughput demand of the wireless link can be reduced. The control parameters specify the modality of stimulation, the amplitude, shape, duration and period of the current output for stimulation, as well as the gain, bandwidth and sampling rate for recording. Since the width and period of the optical pulses can be two orders of magnitude higher than those of the electrical stimulation pulses [2,12], the FSM uses two 16-bit counters to control the pulse width and period over a range between $10 \mu$ s and 655.36 $\mathrm{ms}$, with a fine resolution of $10 \mu \mathrm{s}$. The parameters for specifying the pulse width and period are organized in floating-point format [13], where a 4-bit $M$ and a 4-bit $N$ (6bit for the period) are sent via the radio link for each parameter, and the control logic reconstructs the parameter to a 16-bit value as length $=\left((1 . N) * 2^{M}\right)_{\text {binary }}$. The floatingpoint format compresses the bit-length of the parameter values sent over the radio link, without sacrificing the resolution in setting pulse timing. The control logic also includes an 8-byte arbitrary waveform look-up table (LUT), so that the shape of the output current can be programmed by writing the LUT via the radio link, for improving the stimulation efficiency [14], or for measuring bioimpedance for electrode contact detection or surgical guidance [15].

\section{Current Driver}

The current driver provides charge balanced biphasic current pulses in arbitrary waveforms for electrical stimulation, and monophasic pulses for driving the LED arrays. Fig. 3 shows the schematic of the current driver, which 


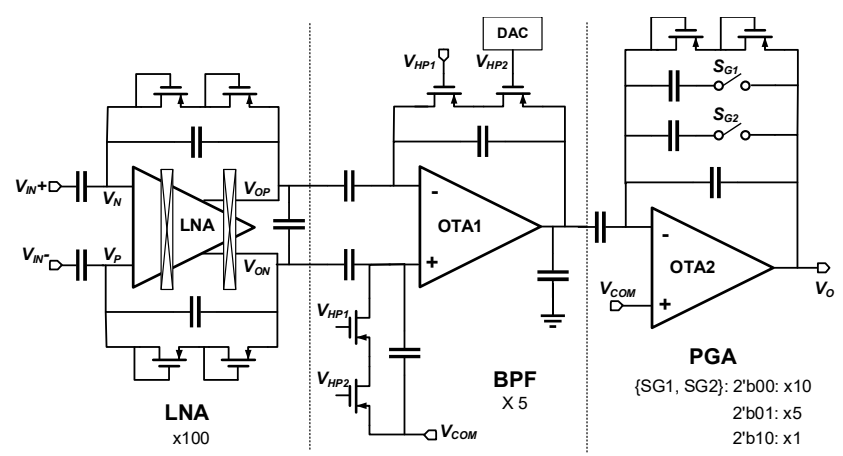

Fig. 4. Implementation of the recording front-end.

consists of a 10-bit current source and an output stage. The output current amplitude is up to $16 \mathrm{~mA}$ to ensure excitation of the engrafted motor neurons that require up to $8 \mathrm{~mW} / \mathrm{mm}^{2}$ light intensity [2]. On the other hand, since the stimulation is localized to innervated motor units, the current amplitude for electrical stimulation, is at $\mu \mathrm{A}$ level. The current driver has 3stage amplitude setting to cover this wide range. The 10-bit current source shown in Fig. 3(a), amplifies a 2-bit reference current, $I_{r e f}$, from $500 \mathrm{nA}$ to $2 \mu \mathrm{A}$, with an 8-bit currentsteering DAC [16], where $I_{\text {ref }}$ is mirrored into 30 branches in two equal weight groups. The 4 most significant bits of the 8bit current setting value are mapped to the 15 branches with a 16:1 ratio via a barrel shifter, and the 4 least significant bits to the 15 branches with a $1: 1$ ratio. The barrel shifters are rotated at every master clock to randomly activate the current branches according the their 4-bit input value, so that nonlinearity caused by mismatch can be randomized. A wideswing current mirror with active feedback, shown in Fig. 3(b), mirrors the output from the current-steering DAC to an $\mathrm{H}-$ bridge to form biphasic current output pulses for electrical stimulation or monophasic pulses for optical stimulation. The ratio of this current mirror can be programmed into $\times 1$ or $\times 32$. Therefore, the equivalent resolution of the current driver is 11bit, and the output current, $I_{\text {out }}$, can be written as

$$
\begin{aligned}
I_{\text {out }}=32^{A[10]} & \times(16 \times A[9: 6]+A[5: 2]) \times(A[1: 0]+1) \\
& \times 500 \mathrm{nA}
\end{aligned}
$$

where $A$ is the 11-bit current setting parameter. In arbitrary waveform generation, $A[9: 2]$ is from the waveform LUT. Transistor M5 in the output branch is a high voltage (HV) transistor, so that the compliance voltage, $V D D H$, can reach up to $18 \mathrm{~V}$, while current source operates at a $1.8 \mathrm{~V}$ supply. Because the threshold voltage of the HV transistor is higher than $1.8 \mathrm{~V}$, the feedback transistors M3 and M4 are supplied at $3.3 \mathrm{~V}$.

The output of the current driver, as shown in Fig.3(b), is multiplexed to 4 cathode contacts and 4 anode contacts. The switch matrix can be configured depending on the application requirement. Fig.3(b) illustrates two configuration examples in the shaded areas. The lower area depicts an all-optical arrangement for 16 individually addressable LEDs, and the upper area illustrates a hybrid optical and electrical arrangement with electrode contacts for electrophysiological recording.

\section{Recording Front-end}

The primary electrophysiological signals of interest are the evoked EMG from innervated motor units and action

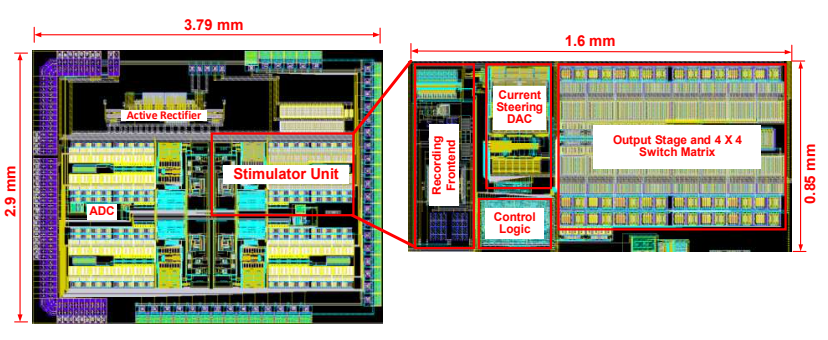

Fig. 5. ASIC layout.

potentials (AP) from the motor nerve. The magnitude of the evoked EMG ranges from 10 s of $\mu \mathrm{V}$ to a few $\mathrm{mV}$, while the frequency can be between $10 \mathrm{~Hz}$ and $5 \mathrm{kHz}$ [17]. The recording front-end consists of three stages, as shown in Fig. 4(a). The first stage is a low-noise amplifier (LNA) with a fixed gain of 100 set by the ratio between the feedback and input capacitors. The second stage is a bandpass filter (BPF) with a passband gain of 5. The upper cut-off frequency is 4 $\mathrm{kHz}$, while the lower cut-off frequency can be programmed by varying the gate voltage of the feedback pseudo-resistor [18] from $1 \mathrm{~Hz}$ for EMG recording, to $100 \mathrm{~Hz}$ for AP recording. The third stage is a programmable gain amplifier (PGA). By setting the switch positions of $S_{G 1}$ and $S_{G 2}$, the PGA gain is variable at 1,5 or 10 . Therefore, the total gain of the recording front-end is from 500 to 5000 .

\section{SIMULATED PERFORMANCE}

The stimulator ASIC is implemented in XFAB $0.18 \mu \mathrm{m}$ HV CMOS technology. Fig. 5 shows the layout of the ASIC. The chip has an area of $11 \mathrm{~mm}^{2}$, where each stimulator unit occupies $1.36 \mathrm{~mm}^{2}$. The power supply for the control logic, current source, recording front-end and SAR ADC is $1.8 \mathrm{~V}$. The output branch of the current driver operates on a high voltage supply $V D D H$ up to $18 \mathrm{~V}$, provided by the DC-DC converter.

Fig. 6 shows examples of four types of output current waveforms for different purposes. Fig. 6(a) shows $50 \mathrm{~Hz}, 50 \%$ duty cycle monophasic current pulses with a peak amplitude of $16 \mathrm{~mA}$ for driving the LEDs for optical stimulation. For this current output, the simulated rms supply current is $576 \mu \mathrm{A}$ at $1.8 \mathrm{~V}$, where $510 \mu \mathrm{A}$ is for the current source output $I_{\text {out }}$, and $8 \mathrm{~mA}$ at $V D D H$. Fig. 6(b) shows charge-balanced biphasic rectangular pulses at $100 \mathrm{~Hz}$ for electrical stimulation. The cathodic phase has a peak amplitude of $8 \mathrm{~mA}$ and a pulse width of $200 \mu \mathrm{s}$. The anodic phase is asymmetrical with the width 8 times longer but the amplitude 8 times smaller. Fig. 6(c) shows biphasic exponential pulses for power efficient electrical stimulation [14]. The peak amplitude of the cathodic phase is $8 \mathrm{~mA}$. The anodic phase is also asymmetrical but maintains charge balance in the same fashion as the pulses in Fig. 6(b). Fig. 6(d) shows a charge-balanced $3.125 \mathrm{kHz}$ sinusoidal current output with a peak amplitude of $128 \mu \mathrm{A}$ for impedance measurement.

The simulated performance of the recording front-end shows the passband gain can be programmed at $\times 5000$ (74 $\mathrm{dB}), \times 2500(68 \mathrm{~dB})$ and $\times 500(54 \mathrm{~dB})$, while rms inputreferred noise over a bandwidth between $1 \mathrm{~Hz}$ and $4 \mathrm{kHz}$ is $7.96 \mu \mathrm{V}$, with a power consumption of $12.2 \mu \mathrm{W}$. The overall performance of the stimulator ASIC is summarized in Table I with comparisons to state-of-the-art integrated optical stimulator designs. 


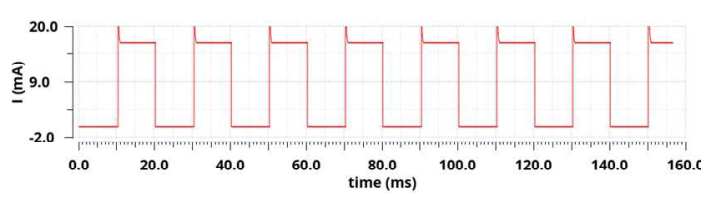

(a)

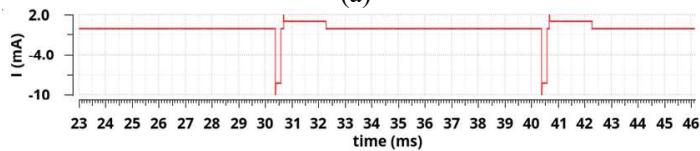

(b)

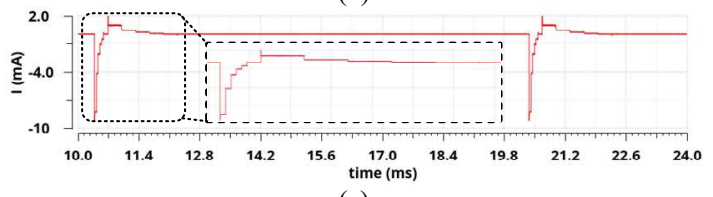

(c)

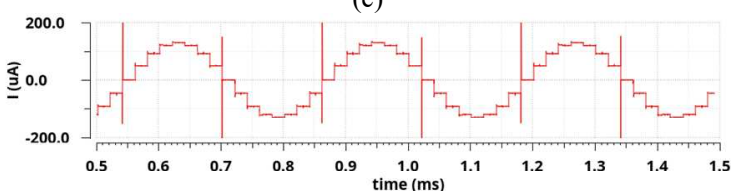

(d)

Fig. 6. Simulated examples of the current driver output waveforms.

\section{CONCLUSION}

A bidirectional multi-channel opto-electro stimulator SoC has been presented. It is capable of both optical and electrical stimulation to up to 64 channels with current waveforms programmable in shape, amplitude and frequency for treating MND using optogenetics and stem cell technology. The chip also has electrophysical recording front-ends with programmable amplifier gain and bandwidth for sensing EMG and action potential, as well as a 10-bit SAR ADC, which can be utilized for closed-loop stimulation control. The chip was designed and fabricated in $0.18 \mu \mathrm{m}$ HV CMOS technology. Simulated performance was reported in this paper. It will be updated with measured results in future.

\section{REFERENCES}

[1] G. Logroscino, et al., "Global, Regional, and National Burden of Motor Neuron Diseases 1990-2016: A Systematic Analysis for the Global Burden of Disease Study 2016," The Lancet Neurology, Vol. 17, Issue 12, 2018, pp. 1083-1097

[2] Bryson JB, et al., "Optical Control of Muscle Function by Transplantation of Stem Cell-Derived Motor Neurons in Mice," Science, 344 (6179), 94-97.

[3] Du J, et al., "Optimal electrical stimulation boosts stem cell therapy in nerve regeneration," Biomaterials, 2018 Oct;181:347-359.

[4] Alex C Thompson, et al., "Hybrid optogenetic and electrical stimulation for greater spatial resolution and temporal fidelity of cochlear activation", 2020 J. Neural Eng. 17056046

[5] G. Gagnon-Turcotte, M. N. Khiarak, C. Ethier, Y. De Koninck and B Gosselin, "A 0.13- $\mu$ m CMOS SoC for Simultaneous Multichannel Optogenetics and Neural Recording," IEEE Journal of Solid-State Circuits, vol. 53, no. 11, pp. 3087-3100, Nov. 2018

[6] R. Ramezani et al., "On-Probe Neural Interface ASIC for Combined Electrical Recording and Optogenetic Stimulation," IEEE Transactions on Biomedical Circuits and Systems, vol. 12, no. 3, pp. 576-588, June 2018

[7] T. Yousefi et al., "An Energy-Efficient Optically-Enhanced HighlyLinear Implantable Wirelessly-Powered Bidirectional Optogenetic Neuro-Stimulator," IEEE Transactions on Biomedical Circuits and Systems, Sep. 2020

[8] H. Lee, K. Y. Kwon, W. Li and M. Ghovanloo, "A Power-Efficient Switched-Capacitor Stimulating System for Electrical/Optical Deep Brain Stimulation," IEEE Journal of Solid-State Circuits, vol. 50, no. 1, pp. 360-374, Jan. 2015
TABLE I. COMPARISON WITH STATE-OF-THE-ART INTEGRATED OPTICAL STIMULATORS

\begin{tabular}{|c|c|c|c|c|}
\hline & [6] & [7] & [8] & "This work \\
\hline Technology & $0.35 \mu \mathrm{m}$ & $0.13 \mu \mathrm{m}$ & $0.35 \mu \mathrm{m}$ & $0.18 \mu \mathrm{m}$ \\
\hline Supply (V) & $\begin{array}{c}3.3(\mathrm{LV}) \\
5(\mathrm{HV})\end{array}$ & 3.3 & \pm 2 & $\begin{array}{c}1.8(\mathrm{LV}) \\
<18(\mathrm{HV})\end{array}$ \\
\hline \multicolumn{5}{|l|}{ Stimulation } \\
\hline $\begin{array}{r}\text { Stimulation } \\
\text { mode }\end{array}$ & Optical & Optical & $\begin{array}{c}\text { Optical } \\
\text { / Electrical }\end{array}$ & $\begin{array}{c}\text { Optical } \\
\text { / Electrical }\end{array}$ \\
\hline Max current & $1.1 \mathrm{~mA}$ & $15 \mathrm{~mA}$ & $4 \mathrm{~mA}$ & $16 \mathrm{~mA}$ \\
\hline $\begin{array}{r}\text { Current } \\
\text { resolution }\end{array}$ & 8-bit & 3-bit & 5-bit & 11-bit \\
\hline Pulse rate & $50 \mathrm{kpps}$ & - & $244 \mathrm{pps}$ & $2-50$ kpps \\
\hline $\begin{array}{r}\text { Pulse timing } \\
\text { resolution }\end{array}$ & - & - & 5-bit & 16-bit \\
\hline $\begin{array}{r}\text { Current } \\
\text { pattern }\end{array}$ & $\begin{array}{l}\text { Biphasic } \\
\text { rectangular }\end{array}$ & Arbitrary & $\begin{array}{c}\text { Biphasic } \\
\text { exponential }\end{array}$ & $\begin{array}{c}\text { Biphasic } \\
\text { arbitrary }\end{array}$ \\
\hline $\begin{array}{r}\text { No. of } \\
\text { channels }\end{array}$ & 6 & 2 & 16 & $16 \times 4$ \\
\hline \multicolumn{5}{|l|}{ Recording } \\
\hline Amp gain & $50 \mathrm{~dB}$ & $48.3 \mathrm{~dB}$ & N/A & $74 \mathrm{~dB}$ \\
\hline Bandwidth & $\begin{array}{c}0.02-830 \\
\mathrm{~Hz}\end{array}$ & $3-1 \mathrm{k} \mathrm{Hz}$ & - & $\begin{array}{c}1-4 \mathrm{k} \mathrm{Hz} \\
100-4 \mathrm{k} \mathrm{Hz} \\
\end{array}$ \\
\hline $\begin{array}{r}\text { Input-referred } \\
\text { noise }\left(\mu V_{\text {rms }}\right)\end{array}$ & 2.1 & 7.79 & - & 7.96 \\
\hline Noise BW & $0.1-300 \mathrm{~Hz}$ & $3-1 \mathrm{k} \mathrm{Hz}$ & & $1-4 \mathrm{k} \mathrm{Hz}$ \\
\hline Amp power & $9.8 \mu \mathrm{W}$ & $160 \mu \mathrm{W}^{\mathrm{a}}$ & - & $12.2 \mu W$ \\
\hline ADC res. & 10-bit & 8-bit & - & 10-bit \\
\hline ADC power & $31.68 \mu \mathrm{W}$ & - & - & $16.2 \mu W$ \\
\hline
\end{tabular}

*Parameters are simulated values.

a Power at the system level without stimulation.

[9] Y. Jia and M. Ghovanloo, "Towards a mm-Sized Free-Floating Wireless Implantable Opto-Electro Stimulation Device," 2019 IEEE Biomedical Circuits and Systems Conference (BioCAS), Nara, Japan, 2019, pp. 1-4

[10] J. Charthad et al., "A mm-Sized Wireless Implantable Device for Electrical Stimulation of Peripheral Nerves," IEEE Transactions on Biomedical Circuits and Systems, vol. 12, no. 2, pp. 257-270, April 2018

[11] M. Schormans, V. Valente and A. Demosthenous, "Practical Inductive Link Design for Biomedical Wireless Power Transfer: A Tutorial," IEEE Transactions on Biomedical Circuits and Systems, vol. 12, no. 5, pp. 1112-1130, Oct. 2018

[12] V. Giagka, C. Eder, N. Donaldson and A. Demosthenous, "An Implantable Versatile Electrode-Driving ASIC for Chronic Epidural Stimulation in Rats," IEEE Transactions on Biomedical Circuits and Systems, vol. 9, no. 3, pp. 387-400, June 2015

[13] D. Jiang, A. Demosthenous, T. Perkins, X. Liu and N. Donaldson, "A Stimulator ASIC Featuring Versatile Management for Vestibular Prostheses," IEEE Transactions on Biomedical Circuits and Systems, vol. 5, no. 2, pp. 147-159, April 2011

[14] Warren M.Grill, "Model-based analysis and design of waveforms for efficient neural stimulation", Prog Brain Res, 222 (2015), pp. 147-162

[15] C. K. Giardina, E. S. Krause, K. Koka and D. C. Fitzpatrick, "Impedance Measures During in vitro Cochlear Implantation Predict Array Positioning," IEEE Transactions on Biomedical Engineering, vol. 65 , no. 2, pp. 327-335, Feb. 2018,

[16] D. Jiang, Y. Wu and A. Demosthenous, "Hand Gesture Recognition Using Three-Dimensional Electrical Impedance Tomography," IEEE Transactions on Circuits and Systems II: Express Briefs, vol. 67, no. 9, pp. 1554-1558, Sept. 2020

[17] Arnold WD, Sheth KA, Wier CG, Kissel JT, Burghes AH, Kolb SJ., “ Electrophysiological Motor Unit Number Estimation (MUNE) Measuring Compound Muscle Action Potential (CMAP) in Mouse Hindlimb Muscles," J Vis Exp. 2015;(103):52899, 2015 Sep 25.

[18] Y. Liu, S. Luan, I. Williams, A. Rapeaux and T. G. Constandinou, "A 64-Channel Versatile Neural Recording SoC With Activity-Dependent Data Throughput," IEEE Transactions on Biomedical Circuits and Systems, vol. 11, no. 6, pp. 1344-1355, Dec. 2017 
